\title{
Pérdidas de Suelo Bajo Diferentes Arreglos Productivos en el Corredor Biológico entre Parques Nacionales Naturales Puracé y Cueva de los Guácharos
}

\author{
Losses of Soil Under different productive \\ arrangements in the Biological Corridor in \\ National Nature Park and Guacharos Cave
}

\author{
Armando Torrente Trujillo ${ }^{1}$
}

\begin{abstract}
Resumen
Se evaluaron las pérdidas de suelo en parcelas de escorrentía mediante la Ecuación USLE y la aplicación del modelo Epic (Environmental Policy Integrated Climate). Para los propósitos se seleccionaron tres fincas, localizadas en el municipio de Palestina (veredas Jericó y Pinos) y Pitalito (Finca Marengo) con manejo tradicional y alternativo de cultivos. Se realizaron los análisis físicos y químicos de los suelos en las parcelas, se instalaron pluviómetros, estructuras de captación y aforadoras para la medición de sedimentos y escorrentías en cada parcela experimental. En laboratorio se determinó la concentración de los sedimentos totales a través de procesos de filtración y secado. Se estimaron las pérdidas de suelo (ton/ha) para cada evento pluviométrico mediante la medición y aplicación de los modelos mencionados. Las parcelas con manejo alternativo mostraron perdidas de suelo significativamente menores comparadas con las parcelas tradicionales.
\end{abstract}

Palabras Claves: Perdidas de Suelo, Erosión del Suelo, Ecuación USLE.

\begin{abstract}
Abstrac
The purpose is to assess the soil loss from runoff plots using Equation USLE and the application of the Epic (Environmental Policy Integrated Climate). Three farms were selected, located in the town of Palestine (Jerico y Pinos) and Pitalito (Marengo farms) with conventional management and alternative crops. Were performed physical and chemical analysis of soils in the plots, rain gauges were installed, intake structures and gauging for measuring sediment and runoff in each plot. In the laboratory is determined the total sediment concentration by filtering and drying processes. Quantified and simulated soil loss (ton/ha) for each rainfall event by measuring and application of the models. Alternative management plots showed significantly lower soil losses compared to conventional plots.
\end{abstract}

Keywords: Losses of Soil, Erosion of the Soil, Equation USLE.

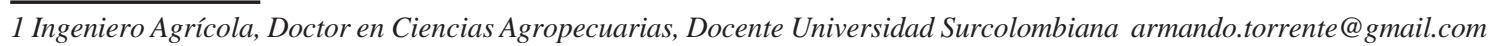




\section{Introducción.}

Las causas, formas y dinámicas de erosión son variadas. La búsqueda de máximos beneficios a corto plazo de la agricultura y la ganadería intensiva se ha convertido en la principal causa de degradación de este recurso natural. Algunas prácticas características de la agricultura intensiva están propiciando un aumento de la presión y degradación de los suelos. Estos procesos degenerativos del suelo, especialmente en las zonas de ladera, tienen un amplio efecto en las cuencas del territorio nacional donde se presentan altos porcentajes de degradación por erosión, remoción en masa y/o sedimentación, entre las cuales se destaca la Cuenca del Río Magdalena con valores muy altos de degradación.

El Corredor Biológico entre los Parques Nacionales Naturales Puracé y Cueva de los Guácharos - $\mathrm{CB}$ - se ha considerado como uno de sus ejes centrales la estrategia de sistemas productivos sostenibles, como una forma de contribuir a la conservación de la diversidad biológica y cultural, y a su vez al mejoramiento de la calidad de vida de las comunidades, mediante el conocimiento y manejo de los ecosistemas naturales y los sistemas de producción agropecuaria. El objetivo es determinar las pérdidas de suelo en ocho (8) arreglos productivos implementados en el Corredor Biológico, y evaluar comparativamente la efectividad de sistemas productivos convencionales y alternativos con prácticas de conservación de suelos.

\section{Metodología.}

La investigación se desarrolló en los municipios de Palestina y Pitalito (Huila). Se construyeron parcelas de escorrentía, las cuales representan el manejo alternativo de los cultivos implementados en el Corredor Biológico y el manejo tradicional, esto con el fin de cuantificar y comparar las pérdidas de suelos en los diferentes arreglos (Figura1).

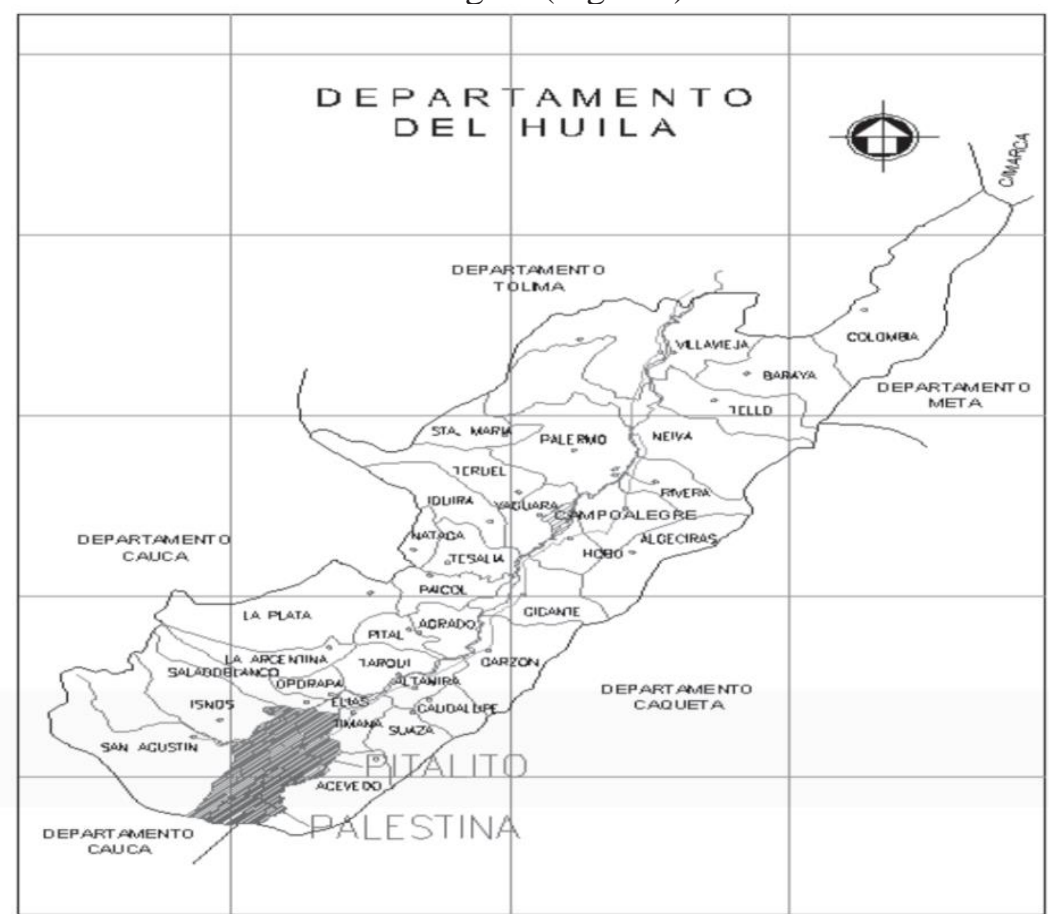

Figura 1. Localización de área experimental al sur del Departamento del Huila. 
Para el establecimiento de la parcela se seleccionaron arreglos productivos sostenibles propuestos por el Corredor Biológico y los tradicionales de los agricultores (Tabla 1, Figura 2).

Tabla 1. Sistemas productivos en el Corredor Biológico

\begin{tabular}{|c|c|c|}
\hline Sistemas Productivos & Tradicional & Alternativo \\
\hline Café & convencional & bajo sombra \\
\hline Mora & convencional & barreras vivas \\
\hline Ganadería & extensiva & Semiestabulada \\
\hline Cultivos semestrales & labranza convencional & labranzas cero \\
\hline
\end{tabular}

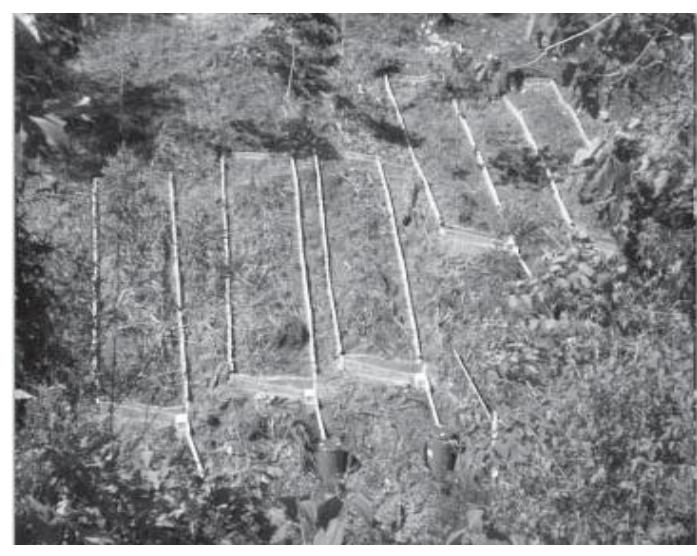

Figura 2.

a) Parcelas en la Vereda Pinos

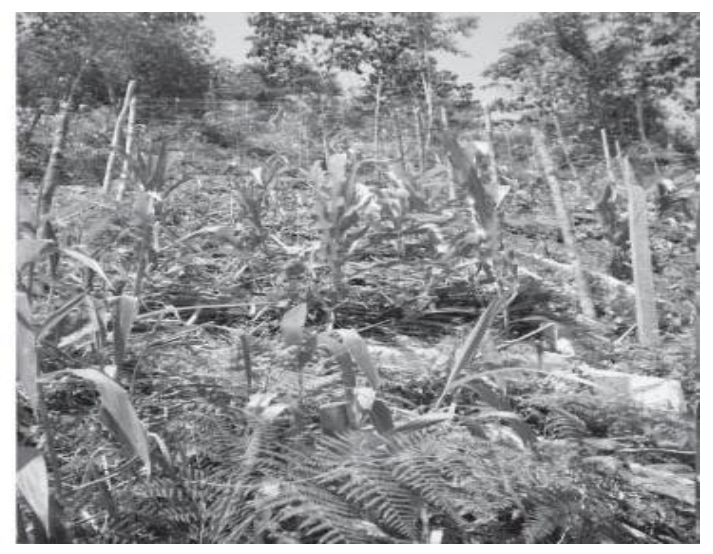

b) Tratamiento labranza cero

La estructura del experimento constó de 11 tratamientos que incluyen parcela desnuda, mora, café, cultivo semestral y ganadería, con 3 repeticiones teniendo como testigo la parcela desnuda en cada localidad, el diseño es en bloques completos al azar. La unidad experimental es la parcela de escorrentía y la variable de respuesta es la producción de sedimentos en la parcela bajo tratamiento de cobertura, uso y manejo convencional o alternativo.

En cada finca donde se ubicaron las parcelas se realizaron mediciones del área y la pendiente del terreno, el muestreo y el análisis físico y químico del suelo, así como pruebas de conductividad hidráulica (pozo barrenado) e infiltración (anillos concéntricos) del suelo. Se impartió capacitación a los agricultores en las distintas parcelas de escorrentía para la recolección y manipulación de las muestras, el registro de información y el debido mantenimiento de las parcelas.

Se realizaron lecturas de precipitación diaria y se recolectó después de cada evento pluvial, una muestra de $100 \mathrm{ml}$ de la escorrentía de las parcelas para su análisis en el laboratorio mediante el método del papel filtro. En cada estructura colectora de escurrimiento fue medido el volumen total de agua y los sedimentos transportados. La cantidad total de sedimentos por parcela permitió proyectar la pérdida de suelo a una hectárea.

El índice de Erosividad (EI30) del evento pluvial se obtuvo de la fórmula de Smith y Wischmeier (1963) citado por USDA 1991, este parámetro es igual al producto de la energía de las gotas de lluvia y su intensidad máxima en 30 minutos. El índice de Erodabilidad (K) se determinó por la ecuación de Paulet. El factor LS se obtuvo aplicando la ecuación de Wischmeier y Smith (1978) citado por Foster et al. 1982. Para el factor C se aplicaron los índices propuestos por Roose (1977) y García (1992) citado por Benson et al. 1989. El factor de prácticas de conservación se estableció según Morgan 1997 citado por Rivera 1999. 


\section{Resultados.}

Los suelos en la parcelas de Jericó y Pinos son de textura franca con baja densidad aparente y alta retención de humedad, estos suelos presentan minerales con estructuras amorfas y alófana cuyo origen son las cenizas volcánicas, corresponden a suelos en evolución, constituido en mayor proporción por minerales no cristalinos y difieren de las características físicas de los suelos de Marengo (Tabla 2).

Tabla 2. Características físicas de los suelos en el Corredor Biológico

\begin{tabular}{|c|c|c|c|c|c|}
\hline \multirow{2}{*}{ Sitio } & Horizonte & Textura & $\begin{array}{c}\text { Densidad } \\
\text { Aparente }(\mathbf{g} / \\
\mathbf{c m} 3)\end{array}$ & $\begin{array}{c}\text { Densidad } \\
\text { Real (g/cm3) }\end{array}$ & Humedad\% \\
\hline \multirow{2}{*}{$\begin{array}{c}\text { JERICÓ } \\
\text { Parcela con cultivo de Mora }\end{array}$} & $\mathrm{A}$ & $\mathrm{F}$ & 0.84 & 1.61 & 69.15 \\
\cline { 2 - 6 } & $\mathrm{AB}$ & $\mathrm{F}$ & 0.90 & 2.20 & 35.00 \\
\cline { 2 - 6 } & $\mathrm{B}$ & $\mathrm{F}$ & 0.77 & 2.31 & 46.70 \\
\hline \multirow{2}{*}{$\begin{array}{c}\text { JERICÓ } \\
\text { Parcela con cultivo de Café }\end{array}$} & $\mathrm{A}$ & $\mathrm{F}$ & 0.65 & 1.10 & 43.18 \\
\cline { 2 - 6 } & $\mathrm{B}$ & $\mathrm{F}$ & 0.85 & 2.24 & 25.64 \\
\hline \multirow{2}{*}{\begin{tabular}{c} 
PINOS \\
\cline { 2 - 6 }
\end{tabular}} & $\mathrm{A}$ & $\mathrm{F}$ & 0.68 & 2.21 & 49.25 \\
\cline { 2 - 6 } & $\mathrm{B}$ & $\mathrm{ArL}$ & 1.37 & 2.47 & 20.31 \\
\hline $\begin{array}{c}\text { Parcela con ganadería } \\
\text { extensiva }\end{array}$ & $\mathrm{A}$ & $\mathrm{FAr}$ & 1.42 & 2.40 & 2.47 \\
\cline { 2 - 6 } & $\mathrm{C}$ & $\mathrm{Ar}$ & 1.53 & 2.45 & 6.00 \\
\hline
\end{tabular}

F franco, FArL franco arcillo limoso, ArL arcillo limoso, FAr franco arcilloso, Ar arcilloso.

En Marengo el 95\% de los eventos pluviales se clasificaron como suaves, mientras que en Pinos y Jericó la proporción de eventos pluviales fuertes y severos fue del 13 y $10 \%$ respectivamente (Figura 3).

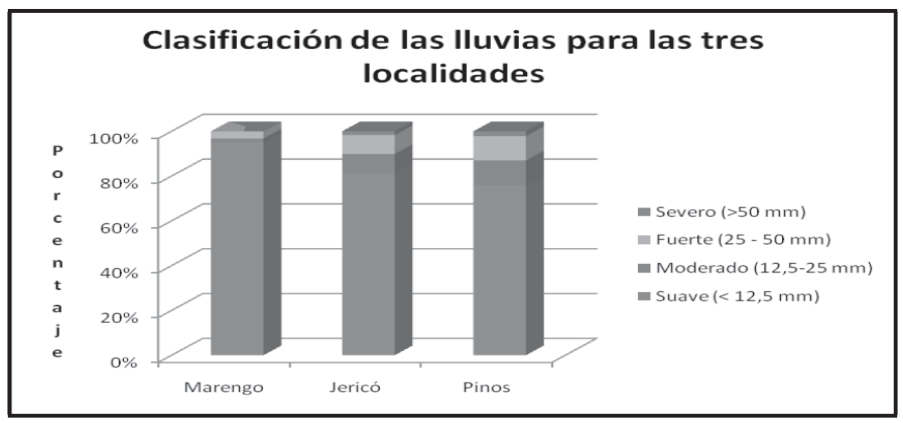

Figura 3. Clasificación de las lluvias en las áreas experimentales

Las mayores pérdidas ocurrieron en la parcela de suelo desnudo en la localidad de Jericó, con dos picos de eventos pluviales cuyas pérdidas fueron 27 y $39 \mathrm{~kg} / \mathrm{ha}$, estos eventos coincidieron con las labores previas de limpieza; la pérdida total alcanzó un valor de $411 \mathrm{~kg} / \mathrm{ha}$ en el periodo de medición. 
En general, el tratamiento de mora con barreras vivas presentó los niveles más bajos de pérdidas $(129 \mathrm{~kg} / \mathrm{ha})$, cantidad que representa la mitad de la pérdida de suelo ocasionada en el tratamiento con mora convencional ( $249 \mathrm{~kg} / \mathrm{ha})$, explicado esto en el efecto que tienen dichas barreras en el arrastre de partículas del suelo.

Al comparar los dos tratamientos con café se comprueba que la menor pérdida de suelo se registra en el tratamiento café bajo sombra $(182 \mathrm{~kg} / \mathrm{ha})$, en la gráfica se observan picos que corresponden a eventos de alta precipitación, no obstante el efecto es más acentuado en la línea que representa el cultivo de café tradicional (Figura 4 y Figura 5).

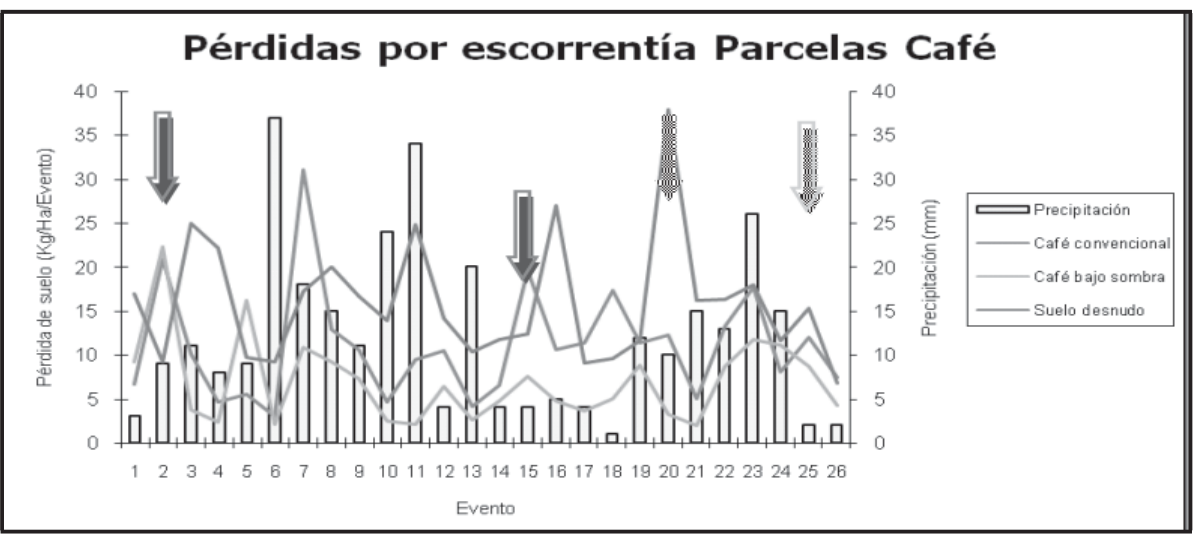

Figura 4. Pérdidas por escorrentía en parcelas de café

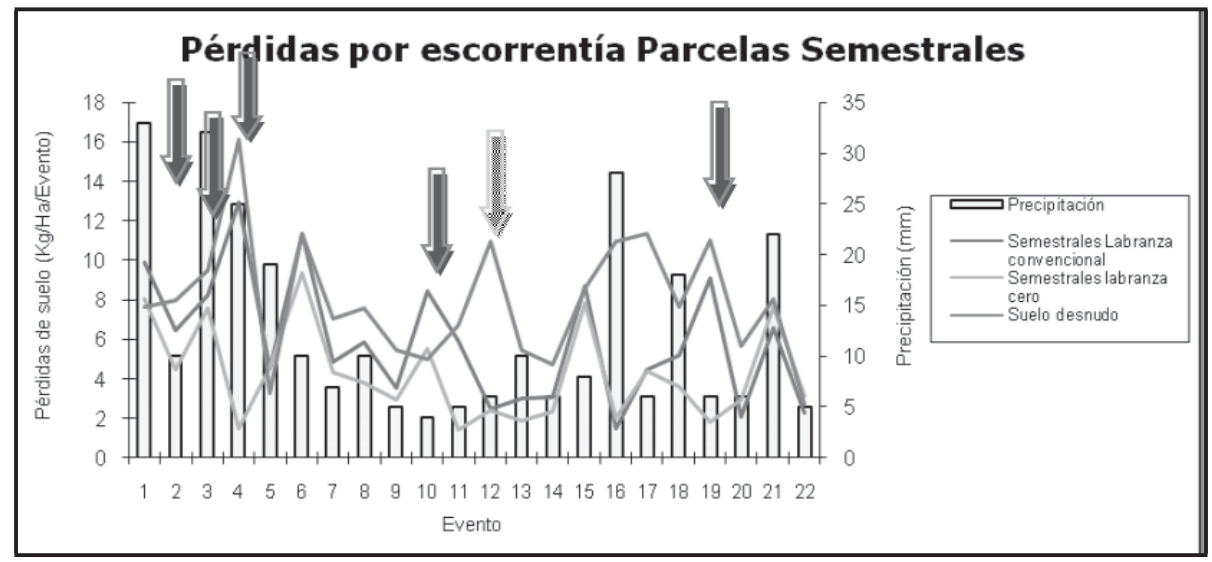

Figura 5. Pérdidas por escorrentía en parcelas de cultivos semestrales

Las mayores pérdidas ocurrieron en cultivos semestrales con labranza convencional (130 $\mathrm{kg} / \mathrm{ha} / 4$ meses), mientras con labranza cero fue de $94 \mathrm{~kg} / \mathrm{ha} / 4$ meses, con picos en labranza convencional de 12.95 y $11.33 \mathrm{~kg} / \mathrm{ha} /$ evento para precipitación de 25 y $10 \mathrm{~mm}$ respectivamente. Valores críticos se observaron en el establecimiento del cultivo por labores de quema y remoción de la cobertura vegetal en el tratamiento de labranza convencional, con valores similares a parcela desnuda. Por el contrario, en este mismo evento la pérdida en labranza cero fue de $1,7 \mathrm{~kg} /$ ha por el mantenimiento de la cobertura y la presencia de residuos de barbecho sobre el suelo. 
En el tratamiento ganadería extensiva resultaron altas pérdidas de suelo coincidiendo con la ocurrencia de eventos pluviales y prácticas de limpieza de los potreros. Durante la permanencia del animal en las parcelas, las pérdidas fueron similares a las de periodos de descanso; estos valores no alcanzan a los de ganadería extensiva debido al proceso de recuperación de la cobertura en los periodos de descanso y al efecto sobre la protección del suelo (Ver Tabla 3).

Tabla 3. Pérdidas de suelo en los distintos tratamientos

\begin{tabular}{|c|l|c|}
\hline Sitios & \multicolumn{1}{|c|}{ Tratamiento } & Erosión (ton/ha-año) \\
\hline \multirow{4}{*}{ Jericó } & Parcela desnuda & 1.64 \\
\cline { 2 - 3 } & Mora Convencional & 0.99 \\
\cline { 2 - 3 } & Mora barreras vivas & 0.51 \\
\cline { 2 - 3 } & Café Convencional & 1.14 \\
\cline { 2 - 3 } & Café bajo sombra & 0.73 \\
\hline \multirow{3}{*}{ Pinos } & Parcela desnuda & 1.50 \\
\cline { 2 - 3 } & Cultivos semestrales & 0,53 \\
\hline & Cahranza convencional & 0.37 \\
\cline { 2 - 3 } & Cultivos semestrales labranza cero & 1.20 \\
\hline \multirow{4}{*}{ Marengo } & Parcela desnuda & 0.33 \\
\cline { 2 - 3 } & Ganadería extensiva & 0.31 \\
\cline { 2 - 3 } & Ganadería semiestabulada & \\
\hline
\end{tabular}

\section{Conclusiones}

Existen diferencias significativas a un nivel del 5\% entre los tratamientos convencionales y los tratamientos alternativos propuestos, lo cual permite concluir que las labores productivas asociadas a prácticas de conservación en el manejo de las áreas agrícolas son importantes en la sostenibilidad del suelo.

Las coberturas más susceptible a erosión son aquella con cultivos semestrales convencionales cuyo factor $\mathrm{C}$ es 0.39 , y por el contrario las coberturas protectoras del suelo (menor índice) son las prácticas de café bajo sombra y mora con barreras vivas.

La mayor erosión se presenta en el cultivo de café convencional (1.14 tn/ha/año Vs 0.73 tn/ha/año en Café bajo sombra), atribuida principalmente al manejo de la cobertura.

La cobertura densa es la práctica más eficiente de conservación de suelos y aguas como lo muestran las parcelas del cultivo de mora; la erosión para el cultivo de mora convencional fue de $0.99 \mathrm{tn} / \mathrm{ha}$-año contra $0.51 \mathrm{tn} / \mathrm{ha}$-año del cultivo de mora con barreras vivas.

En ganadería extensiva la erosión fue similar al de ganadería semiestabulada, 0.33 y $0.31 \mathrm{tn} / \mathrm{ha}$ año respectivamente, demostrando que el efecto de la intensidad de la lluvia combinado con las prácticas de manejo en los cultivos asociados al grado de cobertura, tienen influencia importante en la pérdida de suelo por escorrentía en las zonas de ladera.

\section{Agradecimientos.}

A la ONF Andina, Cormagdalena, CAM y FFEM, en especial Al Ingeniero Camilo Augusto Agudelo, Director Técnico del Proyecto Corredor Biológico y a los auxiliares de investigación Adriana Vargas y Astrid Lorena Dussán. Así mismo a los agricultores de las Veredas Jericó y Pinos en el municipio de Palestina - Huila, como a los funcionarios de la Finca Marengo en el municipio de Pitalito - Huila. 


\section{Referencias Bibliográficas}

1. Benson, V., Rice, O., Dyke, P., Williams, J., y Jones, C. 1989. Conservation impact on crop productivity for the life of a soil J. Soil and Water Conservation. Vol. 44. p. 600-604.

2. Foster G.R., Moldenhauer W.C. y Wischmeier W.H. 1982. Transferability of US technology for prediction and control of erosion in the tropics. En: Soil Erosion and Conservation in the Tropics, Special Publication 43. Amer. Soc. of Agronomy. p. 135-149.

3. Rivera, H. 1999. Susceptibilidad y predicción de la erosión en suelos de ladera de la zona cafetera colombiana. Colombia. $50 \mathrm{p}$.

4. USDA. 1991. Predicting Soil Erosion by Water - A Guide to Conservation Planning with the Revised Universal Soil Loss Equation (RUSLE). Draft Handbook for limited circulation, Departamento de Agricultura de los Estados Unidos, Washington DC. 